\title{
Asymmetric vortex shedding flow past an inclined flat plate at high incidence
}

\author{
K. M. Lam*, M. Y. H. Leung \\ Department of Civil Engineering, The University of Hong Kong, \\ Pokfulam Road, Hong Kong, China
}

Submitted to: $\quad$ European Journal of Mechanics, B - Fluids

(Final version)

Number of pages: $\quad 24$

Number of figures: 11

Number of manuscript submitted: 1

Corresponding author:

Dr. K. M. Lam

Department of Civil Engineering

The University of Hong Kong

Pokfulam Road

Hong Kong, China

Tel: (852) 28591975

Fax: (852) 25595337

E-mail: kmlam@hku.hk 


\title{
Asymmetric vortex shedding flow past an inclined flat plate at high incidence
}

\author{
K. M. Lam*, M. Y. H. Leung \\ Department of Civil Engineering, The University of Hong Kong
}

\begin{abstract}
This paper reports an experimental investigation of the vortex shedding wake behind a long flat plate inclined at a small angle of attack to a main flow stream. Detailed velocity fields are obtained with particle-image velocimetry (PIV) at successive phases in a vortex shedding cycle at three angles of attack, $\alpha=20^{\circ}$, $25^{\circ}$ and $30^{\circ}$, at a Reynolds number $R e \approx 5,300$. Coherent patterns and dynamics of the vortices in the wake are revealed by the phase-averaged PIV vectors and derived turbulent properties. A vortex street pattern comprising a train of leading edge vortices alternating with a train of trailing edge vortices is found in the wake. The trailing edge vortex is shed directly from the sharp trailing edge while there are evidences that the formation and shedding of the leading edge vortex involve a more complicated mechanism. The leading edge vortex seems to be shed into the wake from an axial location near the trailing edge. After shedding, the vortices are convected downstream in the wake with a convection speed roughly equal to 0.8 the free-stream velocity. On reaching the same axial location, the trailing edge vortex, as compared to the leading edge vortex, is found to possess a higher peak vorticity level at its centre and induce more intense fluid circulation and Reynolds stresses production around it. It is found that the results at the three angles of attack can be collapsed into similar trends by using the projected plate width as the characteristic length of the flow.
\end{abstract}

(Keywords: inclined flat plate / vortices / PIV) 


\section{Introduction}

Flow around a two-dimensional bluff body is a basic fluid mechanic topic being extensively studied. The flow is also relevant to numerous engineering applications. Key mean properties of bluff-body flow include flow separation, wake formation and development of drag and lift forces. Dynamic features of the flow such as vortex shedding and fluctuating velocity fields are equally important and lead to flow-induced vibration, noise generation and turbulence mixing. The long circular cylinder and the flat plate or the square cylinder normal to the flow are representative examples of bluff-body flows with non-fixed and fixed-point separation. They have been studied most extensively among other body shapes in experimental and numerical studies. The wake behind this class of bluff bodies possesses a symmetrical time-averaged mean pattern while the instantaneous flow is dominated by periodic coherent patterns due to vortex shedding. Flow separates on either side of the cylinder or at the two edges of the flat plate. The two shear layers of equal strengths roll up alternatively into large-scale vortices which are then shed into the wake in the form of a vortex street. Dynamics of the flow and the evolution of the vortex street have been studied experimentally with the phase averaging technique. Notable examples include Cantwell and Coles [1] on flow over a circular cylinder, Leder [2] on flow past a flat plate, and Lyn and Rodi [3] on flow around a square cylinder. In these studies, phase-averaged velocity fields in the wake were obtained with a flying hot wire or laser Doppler anemometer (LDA). The results at successive phases in a vortex shedding cycle showed the development and dynamics of the equal-strength but opposite-signed alternating vortices in the vortex street.

The symmetrical time-averaged mean wake pattern behind the bluff body can be disturbed by the introduction of asymmetric flow conditions. Flow past a rotating circular cylinder is one example. Numerical investigations have showed that the wake is deflected to one side by the constant rotation of the cylinder and an asymmetric wake pattern was observed [4,5]. A lift force is produced on the rotating cylinder from the Magnus effect. At relatively low rotational speeds of the 
cylinder, alternate vortex shedding from two sides of the cylinder still occurs. The alternatively shed vortices of opposite-signed vorticity are found to possess similar strengths in the wake. In the near-surface flow, however, the vorticity on the side of the cylinder moving against the free-stream is found to be more dominant than the vorticity of the opposite sign on the other side of the cylinder where the velocity difference across the separation shear layer is smaller [4]. These observations suggest that an asymmetric flow condition may lead to some degree of asymmetry in vortex shedding and vortex pattern in the wake.

A flat plate inclined to a free-stream at high incidence is another example where the wake flow is asymmetric about the wake centreline. Likewise, the plate is under a lift force. Past investigations have showed that over a wide range of angle of attack, vortex shedding occurs from the two edges of an inclined plate, leading to a vortex street in the wake. The early experiment of Fage and Johansen [6] showed that the Strouhal number of vortex shedding scales with the projected width $B$ ' of the plate normal to the incident free-stream. At an angle of attack from $\alpha=30^{\circ}$ to $90^{\circ}$, the Strouhal number has an approximately constant value at $S t^{\prime}=f B^{\prime} / U_{\mathrm{o}}=$ 0.148 ; where $f$ is the vortex shedding frequency and $U_{\mathrm{o}}$ is the free-stream velocity. The more recent study of Knisely [7] also found a nearly constant $S t^{\prime} \approx 0.15$ for $\alpha$ down to $30^{\circ}$ and a sharp increase in $S t^{\prime}$ as $\alpha$ becomes even smaller. It is found that the vortex from the trailing edge dominates the wake of the inclined plate.

Perry and Steiner [8] investigated the flow past a flat plate inclined at $\alpha=$ $45^{\circ}$ using a flying hot-wire and the phase averaging technique. Velocity vector fields and streamline patterns behind the plate have been obtained at 16 successive phases in a vortex shedding cycle. The results revealed the dynamics of the vortices rolled up from the leading and trailing edges of the inclined plate. The vortex rolled up from the leading edge was found to remain attached to the rear of the plate for a longer period of time than the trailing edge vortex. The data appear to the present authors that the leading edge vortex becomes completely detached from the plate and starts its convection in the wake only at a location near the trailing edge. The measurement region covered an axial distance of about $3.5 \mathrm{~B}$ ' from the centre of the plate and no information was available on the vortex dynamics in the wake farther 
downstream. The time-averaged mean velocity vector pattern behind the plate was found to be higher asymmetric with a large recirculation region behind the leading edge. The authors of that investigation attributed this observation to the longer permanence time of the leading edge vortex in the near wake region. In an attempt to resolve whether the shed vortices in the detached wake region possess equal strengths, Lam [9] investigated the flow past an inclined flat plate at $\alpha=30^{\circ}$ using phase-averaged LDA measurements. The results showed that the train of trailing edge vortices have higher vortex strength than the train of leading edge vortices.

The observations of unequal strengths in the two vortex trains behind an inclined plate were questioned in a recent analytical study [10]. On the other hand, numerical experiments were recently reported by Breuer et al. [11,12] on flow over an inclined plate at $\alpha=18^{\circ}$ and the computation results showed clearly that the wake is strongly dominated by the trailing-edge vortices. Asymmetric vortex shedding cycles were found at $S t^{\prime} \approx 0.2$ at that value of $\alpha$. It was also reported that there is no regular shedding motion of the clockwise rotating vortices directly at the leading edge. Instead, shear-layer vortices of the Kelvin-Helmholtz instability type are detected at the leading edge and they develop into a large recirculation region attached to the leeward side of the plate. The eventual detachment of this vortex into the wake is suggested to be dependent on the activities and shedding of the trailing edge vortices.

The aim of this paper is to confirm through detailed experiments whether asymmetric vortex shedding occurs behind a flat plate at a small angle of attack. Experiments are carried out at $\alpha=20^{\circ}, 25^{\circ}$ and $30^{\circ}$. Detailed velocity vectors in the wake are measured with particle image velocimetry (PIV). The phase-lock averaging technique is used to study the development of individual vortices within a shedding cycle $[8,9]$.

\section{Experimental Techniques}

Experiments were carried out in the same wind tunnel as in Lam [9]. The tunnel was of the open-return blow-down type with an octagonal test section of 
width $0.45 \mathrm{~m}$. An aluminium flat plate of width $B=40 \mathrm{~mm}$ was mounted horizontally in the test section as shown in figure 1. It was painted in black to minimise light reflection and it was carefully machined to provide sharp and thin edges so that flow could separate at the tips of the leading edge and the trailing edge even at a small angle of attack down to $\alpha=20^{\circ}$. The main sets of PIV experiments were carried out at a free-stream velocity about $U_{\mathrm{o}} \approx 2 \mathrm{~m} / \mathrm{s}$ and the Reynolds number was $R e=U_{\mathrm{o}} B / v \approx 5,300$. The free-stream flow was uniform, to within $1 \%$, across the middle $90 \%$ of the test section and the turbulence intensity was below 0.01 . The blockage ratio of the plate inclined at $\alpha=20^{\circ}$ was $3.7 \%$. At this angle of attack, the aspect ratio of the plate, that is the ratio of the spanwise length to the windward width $B^{\prime}$, had a very large value about 33 .

In the main sets of experiments, the plate was given controlled vibration in the vertical direction as in Lam [9]. The amplitudes of imposed vibration in all experiments were below 0.012 B'. A mechanical exciter (Brüel \& Kjær) was used to vibrate the plate and a displacement meter was used to record the sinusoidal vertical movement of the plate. The plate was vibrated near its natural vortex shedding frequency and lock-in occurred between vortex shedding and plate movement. Thus, the displacement signal provided a phase reference for the vortex shedding phase in a shedding cycle. As shown previously in Lam [9] as well as from later results, the controlled excitation of the plate improved the regularity of vortex shedding which was essential for good-quality phase-locked PIV measurement of the vortex dynamics. Two-dimensionality of the flow was also enhanced by the controlled excitation [9] and end plates were not installed on the plate.

The PIV system was based on the double-pulse cross-correlation technique [13]. Flow illumination was made with a $2 \times 150 \mathrm{~mJ}$ Nd:YAG laser (Quantel Twins) and a laser sheet generator. The laser sheet cut through a longitudinal section of the wake at mid-span of the plate. A dispersed smoke was used to seed the flow with smoke particles. The smoke was produced from a fog fluid (SAFEX) and was introduced at the inlet of the wind tunnel. Firings of the dual laser pulses were timed at a chosen phase in each vibration cycle of the plate. With the controlled vibration 
to produce lock-in synchronisation between vortex shedding and vertical vibration of the plate movement, PIV snapshots were thus taken at a fixed phase of vortex shedding in each shedding cycle. Within each shedding period $T$, velocity fields were obtained at eight successive phases, at $t=(n / 8) T$ with $n=1,2, \ldots, 8$ (figure 1 ).

Pairs of double-pulsed particle images were captured by a PIV camera (PCO Sensicam) with the maximum resolution of 1280 pixels $\times 768$ pixels. The double pulses were separated by a fraction of a millisecond at which the free-stream velocity would produce a displacement of about 10 pixels. Interrogation windows of 64 pixels $\times 64$ pixels, with a $50 \%$ overlay among windows were used. With some cropping of the images at the edges, there were typically $36 \times 26$ velocity vectors in each PIV snapshot. Vorticity at a grid point was calculated from the circulation around the eight neighbouring grid points.

In the experiments without controlled vibration, a hot wire was placed behind the trailing edge of the inclined plate to detect vortex shedding and its frequency. The hot wire was placed approximately at the location $\left(x / B^{\prime}, y / B^{\prime}\right)=$ $(2.5,-0.75)$, where the coordinates $(x, y)$ are measured from the centre of the plate.

\section{Results and Discussion}

At the largest of the three angles of attack under investigation, that is, at $\alpha=$ $30^{\circ}$, the hot-wire spectrum in the wake of non-vibrating plate shows a sharp spectral peak, showing evidence of periodic vortex shedding from the plate. The Strouhal number of the spectral peak remains at a constant value, $S t^{\prime}=f B^{\prime} / U_{\mathrm{o}}=0.14$, over a range of free-stream velocities from $R e \approx 5,000$ to 25,000 . At the smaller angles of attack, $\alpha=25^{\circ}$ and $20^{\circ}$, however, the spectral peak is not as sharp as one would expect from regular vortex shedding from a two-dimensional bluff body. This observation is particular obvious at low free-stream velocities, at $R e<10,000$. The implications may be that natural vortex shedding from a stationary plate at high incidence is impaired with poor periodicity and poor regularity, perhaps due to increasing degrees of three-dimensionality of the wake [8]. Values of vortex shedding frequency are thus determined from the hot-wire spectra measured at the 
higher free-stream velocities, and they give a fairly constant Strouhal number. The values are $S t^{\prime}=0.15$ at $\alpha=25^{\circ}$; and $S t^{\prime}=0.18$ at $\alpha=20^{\circ}$. A value of $S t^{\prime}=0.2$ has been found in recent numerical experiments on a plate inclined at $\alpha=18^{\circ}[11,12]$. All these findings agree with the earlier observation that vortex shedding scales with the projected width of the inclined plate at an angle of attack higher than $\alpha>$ $20^{\circ}$. Then at even smaller inclination angles, the Strouhal number, based on the projected plate width, become higher than the previous constant value around 0.14 to 0.15 .

Application of controlled vibration of the plate has been shown to enhance the regularity of vortex shedding at small angles of attack [9]. The technique is applied in this study. Figure 2 shows the hot-wire spectra measured behind the trailing edge of the plate at the three angles of attack, $\alpha=20^{\circ}, 25^{\circ}, 30^{\circ}$, when the plate is vibrated at different excitation frequencies. The free-stream velocity is about $2 \mathrm{~m} / \mathrm{s}$ in all experiments $(R e \approx 5,300)$ and the amplitudes of vibration were below $0.012 B$ '. In figure 2 , the excitation frequency $f_{\mathrm{ex}}$ is expressed as a Strouhal number by $S t_{\mathrm{ex}}{ }^{\prime}=f_{\mathrm{ex}} B^{\prime} / U_{\mathrm{o}}$. Lock-in phenomenon is observed at all the three values of $\alpha$ over a wide range of $S t_{\mathrm{ex}}$. The vortex shedding frequency becomes locked on to the vibration frequency of the plate; $S t^{\prime}=S t_{\mathrm{ex}}{ }^{\prime}$. At $\alpha=30^{\circ}$ and $25^{\circ}$, the vortex shedding frequency from the vibrating plate outside the lock-in range is lower than that of the natural vortex shedding frequency of the stationary plate, that is at $S t_{\mathrm{ex}}{ }^{\prime}=$ 0 (figures $2 a, b$ ). At $\alpha=20^{\circ}$, sharp spectral peaks corresponding to regular vortex shedding are clearly observed only when the plate is under lock-in resonance (figure $2 c)$.

Measurement of velocity fields phase-averaged at different phases in a vortex shedding cycle has proved to be a valuable tool to investigate the flow dynamics of vortex shedding in the wake of bluff bodies [1-3,9]. In the present study, controlled vibration is applied to improve the regularity of vortex shedding from a flat plate inclined at a small angle of attack. Flow characteristics in the wake behind the plate with lock-in are expected to represent the typical vortex patterns of natural vortex shedding flow behind a stationary plate during regular shedding cycles. From the results in figure 2, a single value of $S t_{\mathrm{ex}}{ }^{\prime}=0.15$ is chosen for the 
controlled vibration of the inclined plate for all the three angles of attack under which PIV measurements of the wake are made. This excitation frequency is near the middle of the lock-in ranges for the three values of $\alpha$.

The phase-averaged PIV velocity fields, $(\langle U\rangle,\langle V\rangle)$, at $\alpha=20^{\circ}$ are shown in figure 3 for eight successive phases within one vertical vibration cycle of the plate, which is equivalent to one vortex shedding cycle. Each velocity field is the ensemble average of 30 PIV snapshots at the same phase. The phases are denoted by the phase number $n$ which corresponds to the time $t=(n / 8) T$ in each shedding cycle. Within the vibration period $T$, the plate is at its central position at phase 4 and phase 8 . It moves to the upper extreme vertical position at phase 2 and is at its lower extreme position at phase 6 (see figure 1). Vorticity is computed from the velocity fields and the equal-vorticity contour lines are shown in figure 3 as normalised values of $\zeta^{\prime}=\zeta B^{\prime} / U_{\mathrm{o}}$. In addition to normalising the vorticity, the projected plate width is also used to normalise the spatial dimensions of the wake which are measured by $x / B^{\prime}$ and $y / B^{\prime}$.

Even at this high incidence on the flat plate at $\alpha=20^{\circ}$, two trains of vortices of opposite senses of rotation are evident from figure 3. They develop from the leading and trailing edges of the plate and are convected downstream in the wake. Flow visualisation pictures showing two vortex trains of the vortex-street type have been obtained in the wake of a thin hydrofoil under a periodic pitching motion with $\alpha$ oscillating between 0 and $20^{\circ}$ [14]. However, there appears from these pictures different vortex formation mechanisms from the leading edge and from the trailing edge. While the counter-clockwise rotating vortex is formed right at the trailing edge by roll-up of the separation shear layer, the formation of the clockwise vortex on the leading edge does not develop simply from a single roll-up. After flow separates from the leading edge, a number of shear-layer vortices are observed on the upper side of the hydrofoil and only at a location near the trailing edge that these vortices agglomerate to form a leading edge vortex that is shed into the wake. In the wake, the flow visualisation pictures show clearly that the trailing edge vortex train induces more intense fluid circulation than the train of leading edge vortices. 
In the steady flow over an inclined plate at $\alpha=18^{\circ}$, a large-eddy simulation flow computation has also suggested different formation mechanisms between the leading edge vortex and the trailing edge vortex [11]. The sketch in figure 4 shows how these vortices are attached to the leeward side of the plate at an instant when a counter-clockwise rotating vortex has just been shed from the trailing edge of the plate. The sketch is produced from a snapshot in the numerically simulated animation of vortex shedding flow in the large-eddy simulation. Unlike the trailing edge vortex which is rolled up and shed directly from the trailing edge, the formation and detachment of the clockwise rotating vortex involve a more complex mechanism. There are a number of shear-layer vortices, three in figure 4 , developed from the leading edge. It is discussed in Breuer and Jovicic [11] that the large-eddy simulation is capable of reproducing the Kelvin-Helmholtz instability which leads to these shear-layer vortices. The shear-layer vortices extend along the streamwise direction over a portion of the plate width from the leading edge. Only towards the rear end of the plate that a big recirculation region of the clockwise rotating sense is formed from the agglomeration of the shear-layer vortices. This completely formed "leading edge vortex" is already very near to the trailing edge of the plate. Its eventual detachment from the plate is found to be affected by the development of the next counter-clockwise vortex at the trailing edge [11]. The pair of vortices are then convected in the wake as an alternating vortex pair in the vortex street.

In the present PIV result in figure 3, shedding of the positive-vorticity vortex from the trailing edge takes place near phase 6 . This is when the plate is near its lower extreme position. At the previous phase prior to shedding, phase 5, the highest level of vorticity, at $\zeta^{\prime} \approx+2.45$, is found at the centre of this vortex. At these two times, the vortex remains essentially attached to the trailing edge and stays at a location near $x / B^{\prime} \approx 2.3$. Then it is shed from the plate and is being convected downstream in the wake during which it grows in size but the peak vorticity level at its centre drops. At phase 7 , it is centred at $x / B^{\prime} \approx 3.0$ and then at $x / B^{\prime} \approx 3.5$ at phase 8. The peak vorticity value drops to $\zeta^{\prime} \approx+2.2$ and +1.8 correspondingly. Further development of the vortex can be followed from the PIV results at phases 1 to 4 and then as the second counter-clockwise rotating vortex at subsequent phases. 
The space-time trajectory of the centre of the trailing edge vortex is traced out in figure 5. It is evident that shedding of the vortex takes place around at $x / B^{\prime} \approx$ 2.5. When the vortex centre is upstream of this location, the vortex is growing and the centre location shifts downstream only with a very slow speed at about $0.1 U_{\mathrm{o}}$. The vortex is then detached from the plate and shed downstream. Downstream of $x / B^{\prime}>2.5$, the vortex centre moves downstream at a rather steady speed at $U_{\mathrm{c}} \approx 0.8$ $U_{\mathrm{o}}$. A similar value of convection speed $U_{\mathrm{c}}$ at about 0.8 the free-stream velocity has been reported in many two-dimensional bluff body wakes [1].

The PIV results in figure 3 cannot reveal the flow events near the leading edge of the plate but it is evident that at the start of the measurement region at $x / B$ ' $\approx 1.8$, the leading edge vortex has not yet been completely shed or convected into the measurement region at a steady convection speed. Closed contours of negative vorticity concentration connected with the vortex are first observed at the start of the measurement region at phase 1 to phase 2. During this time, its centre location does not noticeably move downstream. Following the earlier discussion of the formation mechanism of the leading edge vortex, this suggests that the vortex is at its stage of a large recirculation region being attached to the upper side of the plate. At phase 3, the leading edge vortex starts to move downstream with a low convection speed. This is evident from the space-time trajectory of the centre of the leading edge vortex shown in figure 5.

The contour plots in figure 3 at all phases suggest that intimate interaction occurs between the development of the leading edge vortex and the next trailing edge vortex to be shed. It is discussed in Breuer and Jovicic [11] that the clockwise rotating vortex has enough space to extend in size only after the preceding trailing edge vortex has been shed and the growth will continue until the next trailing edge vortex is developing. The vorticity contours at phases 1 to 4 support this mechanism. The space-time trajectory in figure 5 shows that the leading edge vortex is eventually shed or detached from the plate at phase 4 to 5 , after which it is convected downstream with a near-constant convection speed at $U_{\mathrm{c}} \approx 0.8 U_{\mathrm{o}}$. The vorticity contours in figure 3 suggest that the shedding of the vortex is strongly dependent on the growth of the trailing edge vortex behind it. From phase 4 to 
phase 6, this growing trailing edge vortex is observed to move upwards to fill into the space behind the leading edge vortex. This observation agrees with the findings of Cantwell and Coles [1] that the shedding of a vortex is aided by the intrusion of the opposite-signed vortex from the other side of the body from behind which cuts off the vorticity supply to the shedding vortex from its shear layer. All the above observations suggest that shedding of the well-established leading edge vortex may be completed only at an axial location near the trailing edge where interaction is possible with the next trailing edge vortex.

Figure 5 shows that the space-time trajectory of the leading edge vortex is almost exactly parallel to that of the trailing edge vortex with a temporal separation of about half a shedding period. This supports that the two trains of vortices form a vortex street pattern in the wake. Upstream of $x / B^{\prime}<2.5$, both trajectories have a much slower speed of convection, further supporting that both the leading edge vortex and the trailing edge vortex are shed at a location near the trailing edge of the inclined plate.

Figure $6 a$ shows the value of the peak vorticity at centres of the vortices as they are convected downstream. In going downstream, the vorticity level drops with the growth in vortex size as well as diffusion of vorticity by turbulent actions. The magnitudes of the negative vorticity levels connected with the leading edge vortex are also plotted for easy comparison with those of the trailing edge vortex. It is clearly evident that at all axial locations in the wake, the trailing edge vortex possesses a consistently higher level of peak vorticity at its centre than the leading edge vortex convected to the same axial location. The previous discussion has suggested that the leading edge vortex is actually shed from a location near the trailing edge. Thus, comparison of peak vorticity levels of the vortices at the same $x$ locations would show the differences between the leading edge vortex and the trailing edge vortex in the development of their vortex strengths after they are shed from the plate and being convected downstream in the wake.

In the introduction to their theoretical work, Zannetti and Iollo [10] raised the concern on the existence of a vortex street comprising two trains of unequalstrength and opposite-signed vortices in the wake behind an inclined plate. As the 
lift on the plate has a steady mean value, the average total circulation associated with the vortex trains is expected to be zero. Figure $6 a$ only shows that the peak vorticity level at the vortex centre is higher for the train of trailing edge vortices. A closer examination of the vortex patterns in figure 3 reveals an obvious difference in vortex sizes as well between the two trains of vortices when they reach the same $x$ location. For instance, the size of the trailing edge vortex located at $x / B^{\prime} \approx 5.5$ at phase 3 , as enclosed by the lowest vorticity contours of $\zeta^{\prime}= \pm 0.2$, is evidently smaller than the size of the leading edge vortex located at the same $x$ location at phase 8 . Thus, the counter-clockwise circulation induced by the trailing edge vortex, with a higher vorticity level at the centre but a smaller vortex size, can have the same magnitude as the clockwise circulation around the leading edge vortex convected to the same $x$ location. The more diffused vorticity levels inside the leading edge vortex can be argued to be a result of its more complicated formation mechanism as suggested previously in figure 4.

If the leading edge vortices were formed right at the leading edge and shed directly into the wake from that location, the comparison of vortex strengths between the two trains of vortices should be made at their axial distances measured respectively from the leading edge and the trailing edge. This extreme scenario is shown in figure $6 b$ which plots the peak vorticity levels of the vortices against the axial distances, $x_{\text {edge }}$, of their centres measured respectively from the corresponding edge of the plate. The vorticity level of the leading edge vortex is now higher when both vortices are within $4 B^{\prime}$ from their respective plate edges. However, farther downstream in the wake, the trailing edge vortex eventually has consistently higher levels of peak vorticity.

The phase-averaged velocity fields and vorticity distributions behind a plate inclined at a slightly larger angle $\alpha=25^{\circ}$ are shown in figure 7. The vortex patterns are rather similar to those at $\alpha=20^{\circ}$ (see figure 3). The roll-up and subsequent shedding of a positive vorticity vortex from the trailing edge are revealed clearly by the vorticity contours. At this angle of attack, shedding of the vortex takes place around phase 4 to phase 5 . Half a shedding period later, near phase 1 , the leading edge vortex is shed into the wake. In the wake, the two trains of shed vortices form 
a vortex street pattern with the trailing edge vortex possessing a higher level of peak vorticity at its centre than the leading edge counterpart at same $x$ locations. This is confirmed by the plot of peak vorticity levels at the vortex centres against $x$ locations which is shown in figure $6 a$. The figure also shows the earlier result at $\alpha=$ $20^{\circ}$ and the result at $\alpha=30^{\circ}$ to be discussed later. It is interesting to note that the downstream decays of vorticity levels as the vortices are convected in the wake for the three values of $\alpha$ are similar when plotted in the non-dimensional form of $\zeta^{\prime}=$ $\zeta B^{\prime} / U_{\mathrm{o}}$ against $x / B^{\prime}$. This further supports that the projected plate width $B^{\prime}$ provides a characteristic length scale for the wake behaviour. The comparison of peak vorticity levels of the two vortices made at same values of $x_{\text {edge }}$ is shown in figure $6 b$ at this value of $\alpha=25^{\circ}$. Similar to the results at $\alpha=20^{\circ}$, when the vortices are within $4 B$ ' from their respective edges of the plate, the clockwise rotating vortex developed from the leading edge has a higher peak vorticity level. However when the vortices are convected farther downstream in the wake, the peak vorticity level of the trailing edge vortex becomes consistently higher than that of the leading edge vortex. The same observation is found for the later result at $\alpha=30^{\circ}$.

The difference in vortex sizes between the two trains of vortices at the same axial location can also be observed clearly at this angle of attack, $\alpha=25^{\circ}$, in figure 7. For example, both located at $x / B^{\prime} \approx 7$, the leading edge vortex at phase 1 covers a larger spatial extent of fluid circulation that the trailing edge vortex at phase 4 . The convection of the two trains of vortices in the wake of the plate is shown in the space-time trajectories of vortex centres in figure 5. Their trajectories are parallel to those at $\alpha=20^{\circ}$. As for the relationship of vortex shedding with the vibration phase of the plate, there are some differences between the two flows. The phase difference is about $\pi / 3$, that is one and a half phase number.

Figure 8 presents the phase-averaged PIV velocity fields at $\alpha=30^{\circ}$. A similar pattern of vortex shedding and dynamics is observed. However, shedding of the trailing edge vortex takes place near phase 2, as compared to phase 6 for $\alpha=20^{\circ}$ (figure 3). The vortex pattern is almost exactly out of phase from that in figure 3 by $\pi$. This phase difference becomes evident from the comparison of space-time 
trajectories of the vortices in figure 5. The trajectory of the trailing edge vortex at $\alpha$ $=30^{\circ}$ is close to that of the leading edge vortex at $\alpha=20^{\circ}$. In flow over a circular cylinder undergoing a lateral vibration, the phase relationship between vortex shedding and cylinder displacement has been found to undergo a phase shift of $\pi$ when the excitation frequency changes past the natural vortex shedding frequency [15]. Here in this study, the excitation frequency of plate vibration is set at $S t^{\prime}=$ 0.15 for all the three values of $\alpha$. Thus, in the experiments at $\alpha=30^{\circ}$, the plate is vibrated at a frequency above its natural vortex shedding frequency; while at $\alpha=$ $20^{\circ}$, the excitation frequency of the plate is below the natural vortex shedding frequency (see figure 2). This may explain the observed phase shift.

As discussed earlier, the peak vorticity levels at the vortex centres in figure 8 follow a similar trend of axial development as those at the other two values of $\alpha$. Downstream developments of the peak vorticity levels found at all the three values of $\alpha$ have been shown in figure 6. All the data are now plotted together in a single graph, in figure $9 a$. The projected plate width $B$ ' and the free-stream velocity $U_{\mathrm{o}}$ are used to normalise the vorticity levels and the axial distances. This results in a reasonably good collapse of the three sets of data onto a single trend of decay of vorticity level with axial distance. This provides a strong support that even for a plate at high incidence, the projected plate width is still the characteristic length of the flow governing many wake behaviours such as the vortex shedding frequency and the present development of vorticity levels inside the vortices.

It has been discussed extensively that the leading edge vortex is actually shed into the wake from an axial location near the trailing edge of the plate. Thus, the normalised vorticity level data, $\zeta^{\prime}=\zeta B^{\prime} / U_{\mathrm{o}}$, for both vortex trains are plotted again in figure $9 b$ against the normalised axial distance, $x_{\text {trailing-edge }} / B^{\prime}$, as measured from the trailing edge of the plate. A better collapse of the data at the three values of $\alpha$ is achieved as compared to figure $9 a$. This provides an indirect evidence to support the argument on the shedding location of the leading edge vortex.

Looking into more details at the vortex patterns in figures 3,7 and 8 , it is noted that the leading edge vortex is always nearer to the next trailing edge vortex following it than the previous trailing edge vortex shed before it. In figure 5, the 
temporal separation between this pair of intimate opposite-signed vortices is always less than, though close to, half a vortex shedding period. It has been discussed earlier that the eventual shedding of a leading edge vortex may be brought about by the upward movement of the trailing edge vortex from behind. This may explain the closer relation between these two vortices. Furthermore, the observation provides evidence to the role of the activities of the trailing edge vortex in affecting the shedding location and shedding instant of the leading edge vortex.

Returning to the results at $\alpha=20^{\circ}$, dynamical properties of the vortices are presented in figure 10 at two phases of the vortex shedding cycle. The phases are phase 2 while the plate moves to its upper extreme position and phase 6 during which the plate is at its lower extreme position. The shed vortices are found to be convected downstream at a speed of at $U_{\mathrm{c}} \approx 0.8 U_{\mathrm{o}}$. To an observer moving with the vortices, the phase-averaged vector fields are thus represented by $\left(\langle U\rangle-U_{\mathrm{c}},\langle V\rangle\right)$. These velocity vectors and the associated streamline patterns are shown in figure 10a. They reveal clearly the fluid circulation induced by the shed vortices in the wake. A comparison of vortex strengths and dynamics can be made between the leading edge vortex at phase 2 and the trailing edge vortex at phase 6 , both of which are centred near $x / B^{\prime} \approx 7.5$ at the two different times in a cycle. The streamline pattern shows that the trailing edge vortex is associated with more intense fluid circulation around it.

The coherent Reynolds stresses produced by the vortices are also shown in figure 10. They are calculated from the coherent velocity fluctuations which are estimated by subtracting the phase-averaged velocities from the time-averaged mean velocities; $u_{\mathrm{c}}=\langle U\rangle-\bar{U}$, and $v_{\mathrm{c}}=\langle V\rangle-\bar{V}$. Figures $10 \mathrm{~b}, 10 \mathrm{c}$ and $10 \mathrm{~d}$ show the Reynolds stresses $v_{\mathrm{c}} v_{\mathrm{c}}, u_{\mathrm{c}} u_{\mathrm{c}}$ and $u_{\mathrm{c}} v_{\mathrm{c}}$, respectively. Typical of a vortex street pattern [1], the alternating vortices jointly produce very high values of $v_{c} v_{c}$ in regions between two vortices (figure $10 \mathrm{~b}$ ) while large values of $u_{\mathrm{c}} u_{\mathrm{c}}$ production are found on two lateral sides of each vortex (figure 10c). Concentrations of high values of Reynolds shear stress, $u_{\mathrm{c}} v_{\mathrm{c}}$, of opposite signs are found at the four corners of each vortex (figure 10d). Comparing the production of $u_{\mathrm{c}} v_{\mathrm{c}}$ in figure $10 \mathrm{~d}$ and the $u_{\mathrm{c}} u_{\mathrm{c}}$ production in figure 10c between the leading edge vortex (phase 2) and the trailing 
edge vortex (phase 6 ) near $x / B^{\prime} \approx 7.5$, it is clearly evident that the trailing edge vortex is stronger and induces a higher level of mixing and turbulence production. For the lateral Reynolds stress, $v_{\mathrm{c}} v_{\mathrm{c}}$, very high production occurs on the front side of the trailing edge vortex (phase 6 in figure 10b). At phase 2, the leading edge vortex induces much lower level of $v_{\mathrm{c}} v_{\mathrm{c}}$ production around its front side. The high level concentration of $v_{\mathrm{c}} v_{\mathrm{c}}$ around its rear side is actually induced by the next trailing edge vortex at $x / B^{\prime} \approx 5$.

The results in figure 10 confirm that the trailing edge vortex in the wake has a higher strength and is associated with more intense dynamical activities than the leading edge vortex convected to the same $x$ location half a shedding cycle later. Similar results are found for $\alpha=25^{\circ}$ and the data are not presented here. For the experiments at $\alpha=30^{\circ}$, there is a phase shift of $\pi$ in the phase relationship between vortex shedding and plate movement relative to that at $\alpha=20^{\circ}$ or $\alpha=25^{\circ}$. The dynamical properties of the vortex trains are shown in figure 11. Due to the phase shift, the patterns of fluid circulation and Reynolds stresses at phase 2 in this figure are similar to those at phase 6 in figure 10. Nevertheless, the same observation can be made that the trailing edge vortex is dynamically more active than the leading edge vortex when compared at the same $x$ location. A comparison can be made between the leading edge vortex around $x / B^{\prime} \approx 6$ at phase 2 and the trailing edge vortex near the same $x$ location at $x / B^{\prime} \approx 6.5$ at phase 6 . The just-shed leading edge vortex at $x / B^{\prime} \approx 3$ (phase 2) and the trailing edge vortex at $x / B^{\prime} \approx 4$ (phase 6 ) form another pair of vortices for comparison. For both pairs, the trailing edge vortex, though at a slightly more downstream location than its leading edge vortex counterpart, is clearly associated with higher production levels of Reynolds stresses.

\section{Conclusions}

The shedding of vortices from an inclined flat plate at high incidence has been investigated experimentally. PIV measurements have been made in the wake at three small angles of attack, $\alpha=20^{\circ}, 25^{\circ}$ and $30^{\circ}$. PIV velocity fields are phaselock captured at eight successive phases in a vortex shedding cycle. The inclined 
plate is given controlled lateral vibration near its natural frequency, at $S t^{\prime}=0.15$, to improve the regularity and periodicity of vortex shedding. Lock-in between plate movement and vortex shedding provides a convenient mean for tracking the phase of vortex shedding. Vorticity distributions computed from the phase-averaged PIV results show clearly the convection of two trains of vortices in the wake of the plate inclined at all the three values of $\alpha$. The two trains of vortices are arranged in a vortex street pattern and are convected downstream in the wake with a fairly constant convection velocity at $U_{\mathrm{c}} \approx 0.8 U_{\mathrm{o}}$. Fluid circulation and Reynolds stress production are induced by the vortices and it is found that the trailing edge vortices are connected with more intense dynamical properties than the leading edge vortices convected to the same $x$ location. The peak vorticity level at the vortex centre is higher for the trailing edge vortex which is, however, smaller in size than the leading edge vortex at the same axial location. At $\alpha=20^{\circ}$, the Strouhal number of vortex shedding, based on $B^{\prime}$, becomes higher than the constant value of $S t^{\prime} \approx$ 0.15 at larger values of $\alpha$. Nevertheless, the convection path, sizes of the vortices and downstream drop of peak vorticity level at the vortex centres exhibit similar trends at the three values of $\alpha$, when normalised by the projected width $B$ ' of the plate. This confirms that $B$ ' remains the characteristic length of the wake behind the flat plate even at very high incidence.

The present experimental results and reviewed numerical results suggest some detailed patterns of the questionable asymmetric wake behind a flat plate at high incidence at $30^{\circ}$ or less. When compared at the same $x$ locations, the trailing edge vortex in the separated wake always possesses more intense vorticity levels but a smaller vortex size than the leading edge vortex. A different way of interpretation of vortex strength comparison will be obtained, however, if the development of the vortices is traced back, respectively, to the leading edge and trailing edge of the plate. Within the first $4 B^{\prime}$ distance from their respective plate edges, the clockwise rotating vortex from the leading edge is found to possess a higher peak vorticity level at its centre than the trailing edge vortex. Farther downstream in the wake, the trailing edge vortex regains the higher peak vorticity level when compared to the leading edge vortex at the same $x_{\text {edge }}$ location. On the 
other hand, if the development of the two trains of vortices is both taken to start from the axial location of the trailing edge, the downstream decay curves of peak vorticity levels at the vortex centres at the three values of $\alpha$ collapse well onto a similar trend. This suggests that the detachment and shedding of the leading edge vortex from the plate is likely to occur at a location near the trailing edge.

There is no velocity data in the region above the inclined plate and near the leading edge but it is discussed from the findings of other studies that the formation of the leading edge vortex involves a different mechanism from that of the trailing edge vortex. This may be because the flat plate inclined at high incidence itself acts as a long after-body shape for flow separation at the leading edge. The shear-layer vortices formed at the leading edge thus extend along the streamwise direction over some axial distances and develop into a big recirculation region. This clockwise vortex remains attached to the leeward side of the plate for some time and its growth seems to be affected by the activities of the vortex formed at the trailing edge. There, at the trailing edge, flow separation is free from the effect of the body of the plate. The trailing edge vortex is formed from simple roll-up of the shear layer and is subsequently shed directly from the roll-up location. Above the plate, however, the clockwise recirculation region can only be shed into the wake as a leading edge vortex from an axial location close to the trailing edge. Its detachment from the plate is largely dependent on the growth of the next trailing edge vortex. The present results show that these two vortices are more closely spaced and more intimate in the vortex street.

The different formation mechanisms of the two trains of vortices are believed to result in the different strengths observed between them in the wake. When compared at the same axial location, the leading edge vortex has undergone a longer birth history than the trailing edge vortex. It is thus more diffused with a lower peak vorticity level at its centre and a larger spatial extent of fluid circulation. In addition to the information on vortex pattern and shedding mechanism, the PIV results in this investigation also provide quantitative information on the dynamical properties of the vortices and will be helpful for validation of future numerical works. 


\section{Acknowledgement}

The investigation was supported by the following research grants awarded by the Research Grants Council of Hong Kong: HKU7006/97E and PolyU1/02C. 


\section{References}

[1] Cantwell B., Coles D., An experimental study of entrainment and transport in the turbulent near wake of a circular cylinder, J. Fluid Mech. 136 (1983) 321374.

[2] Leder A., Dynamics of fluid mixing in separated flows, Phys. Fluids A 3(7) (1991) 1741-1748.

[3] Lyn D.A., Rodi W., The flapping shear layer formed by flow separation from the forward corner of a square cylinder, J. Fluid Mech. 267 (1994) 353-376.

[4] Kang S., Choi H., Lee S., Laminar flow past a rotating circular cylinder, Phys. Fluids 11(11) (1999) 3312-3321.

[5] Chew Y.T., Cheng, M., Luo S.C., A numerical study of flow past a rotating circular cylinder using a hybrid vortex scheme, J. Fluid Mech. 299 (1995) 3571.

[6] Fage A., Johansen F.C., On the flow of air behind an inclined flat plate of infinite span, Proc. R. Soc., London, Ser. A 116 (1927) 170-197.

[7] Knisely C.W., Strouhal numbers of rectangular cylinders at incidence: a review and new data, J. Fluids Struct. 4 (1990) 371-393.

[8] Perry A.E., Steiner, T.R., Large-scale vortex structures in turbulent wake behind bluff bodies. Part 1. Vortex formation processes, J. Fluid Mech. 174 (1987) 233270.

[9] Lam K.M., Phase-locked eduction of vortex shedding in flow past an inclined flat plate, Phys. Fluids A 8 (5) (1996) 1159-1168.

[10] Zannetti L., Iollo A., Passive control of the vortex wake past a flat plate at incidence, Theoret. Comput. Fluid Dynamics 16 (2003) 211-230.

[11] Breuer M., Jovicic N., Separated flow around a flat plate at high incidence: an LES investigation, Journal of Turbulence (http://jot.iop.org) 2 (18) (2001) 115.

[12] Breuer M., Jovicic N., Mazaev, K., Comparison of DES, RANS and LES for the separated flow around a flat plate at high incidence, Int. J. Numer. Meth. Fluids 41 (2003) 357-388. 
[13] Willert C.E., Gharib M., Digital particle image velocimetry, Exp. Fluids 10 (1991) 181-193.

[14] McAlister, K.W., Carr, L.W., Water-tunnel visualizations of dynamic stall, in: Crow D.E., Miller J.A. (Eds.), ASME Symposium on Nonsteady Fluid Dynamics, Winter Meeting, 1979, p.103.

[15] Ongoren, A., Rockwell, D., Flow structure from an oscillating cylinder. Part 1. Mechanisms of phase shift and recovery in the near wake, J. Fluid Mech. 191 (1988) 197-223. 


\section{Figure Captions}

Figure 1. Schematic diagram of experimental set up.

Figure 2. Velocity spectra behind the trailing edge of the plate, at $\left(x / B^{\prime}, y / B^{\prime}\right)=$ $(2.5,-0.75)$, for different excitation frequencies of imposed plate vibration: Spectral density shown in logarithmic scale, frequency axis as $S t^{\prime}=f B^{\prime} / U_{\mathrm{o}}$, and excitation frequency as $S t_{\mathrm{ex}}{ }^{\prime}=f_{\mathrm{ex}} B^{\prime} / U_{\mathrm{o}}$.

Figure 3. Phase-averaged PIV vectors, $(\langle U\rangle,\langle V\rangle)$ and vorticity contours, $\alpha=20^{\circ}$ : Phase number shown inside square brackets. Vorticity contours in $\zeta^{\prime}=$ $\zeta B^{\prime} / U_{\mathrm{o}}= \pm 0.2, \pm 0.3, \pm 0.4, \ldots$

Figure 4. Schematic diagram of vortex formation. Black region: positive vorticity; grey region: negative vorticity.

Figure 5. Space-time trajectories of vortex centre: $\circ \alpha=20^{\circ} ; \diamond \alpha=25^{\circ} ; \Delta \alpha=$ $30^{\circ}$. Solid line: trailing edge vortex; broken line: leading edge vortex. Convection velocity at $U_{\mathrm{c}}=0.8 U_{\mathrm{o}}$ shown.

Figure 6. Downstream drop of peak vorticity levels at vortex centres. Positive $\zeta$ for trailing edge vortex, negative $\zeta$ ' for leading edge vortex, broken curves: magnitude of $\zeta$ ' for leading edge vortex. (a) axial distance from plate centre; (b) axial distance from the plate edges.

Figure 7. Phase-averaged PIV vectors, $(\langle U\rangle,\langle V\rangle)$ and vorticity contours, $\alpha=25^{\circ}$ : Phase number shown inside square brackets. Vorticity contours in $\zeta^{\prime}=$ $\zeta B^{\prime} / U_{\mathrm{o}}= \pm 0.2, \pm 0.3, \pm 0.4, \ldots \ldots$ 
Figure 8. Phase-averaged PIV vectors, $(\langle U\rangle,\langle V\rangle)$ and vorticity contours, $\alpha=30^{\circ}$ : Phase number shown inside square brackets. Vorticity contours in $\zeta^{\prime}=$ $\zeta B^{\prime} / U_{\mathrm{o}}= \pm 0.2, \pm 0.3, \pm 0.4, \ldots \ldots$

Figure 9. Downstream drop of peak vorticity levels at vortex centres: $\circ \alpha=20^{\circ}$; - $\alpha=25^{\circ} ; \Delta \alpha=30^{\circ}$. Positive $\zeta$ ' for trailing edge vortex, negative $\zeta$ ' for leading edge vortex, broken curves: magnitude of $\zeta$ ' for leading edge vortex. (a) axial distance from plate centre; (b) all axial distance from the trailing edge.

Figure 10. Phase-averaged fluid circulation and Reynolds stresses of vortices at phase 2 when the plate is at its uppermost position and at phase 6 when the plate is at lower extreme position, $\alpha=20^{\circ}$ : (a) streamlines and velocity vectors, $\left(\langle U\rangle-0.8 U_{\mathrm{o}},\langle V\rangle\right)$ as appeared to an observer moving with the vortices; (b) contours of $v_{\mathrm{c}} v_{\mathrm{c}} / U_{\mathrm{o}}^{2}$; (c) contours of $u_{\mathrm{c}} u_{\mathrm{c}} / U_{\mathrm{o}}{ }^{2}$; (d) contours of $u_{\mathrm{c}} v_{\mathrm{c}} / U_{\mathrm{o}}{ }^{2}$. Vortices shown by background vorticity regions. Contour values in (b), (c), (d) in steps of 0.02 , broken contours for negative values, zero value contour omitted.

Figure 11. Phase-averaged fluid circulation and Reynolds stresses of vortices at phase 2 and at phase $6, \alpha=30^{\circ}$ : (a) streamlines and velocity vectors, , $\left(\langle U\rangle-0.8 U_{\mathrm{o}},\langle V\rangle\right)$ as appeared to an observer moving with the vortices; (b) contours of $v_{\mathrm{c}} v_{\mathrm{c}} / U_{\mathrm{o}}^{2}$; (c) contours of $u_{\mathrm{c}} u_{\mathrm{c}} / U_{\mathrm{o}}^{2}$; (d) contours of $u_{\mathrm{c}} v_{\mathrm{c}} / U_{\mathrm{o}}^{2}$. Vortices shown by background vorticity regions. Contour values in (b), (c), (d) in steps of 0.01 , broken contours for negative values, zero value contour omitted. 


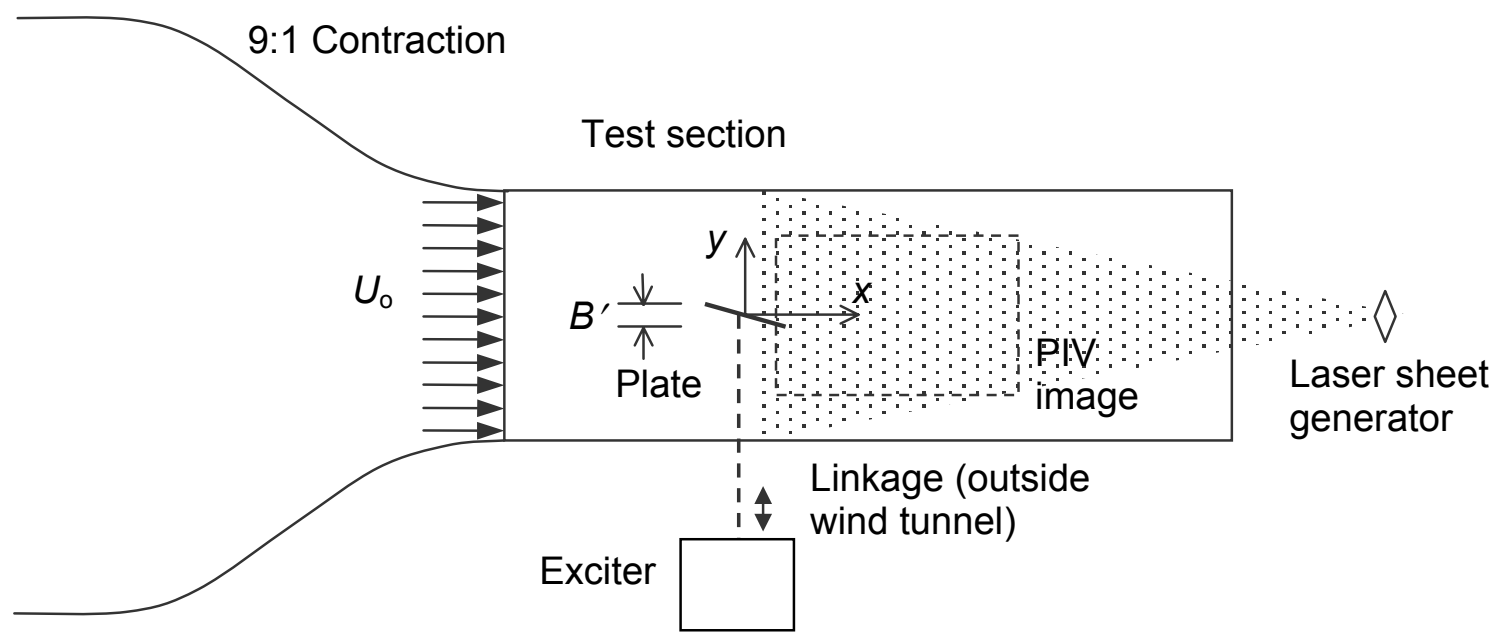

Vertical

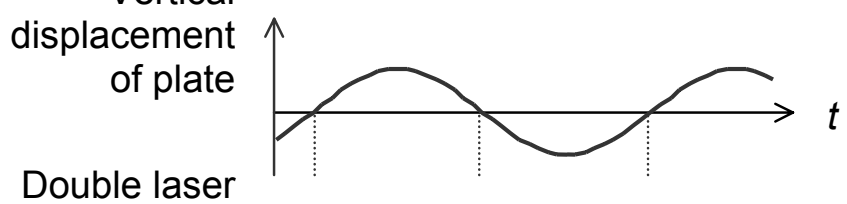

pulses: \|\|\|\|\|\|\|\|\|\|\|\|$\|$

Phase number: $\begin{array}{lllllllllllll}7 & 8 & 1 & 2 & 3 & 4 & 5 & 6 & 7 & 8 & 12\end{array}$ 
Lam and Leung, Figure 2

(a) $\alpha=30^{\circ}$
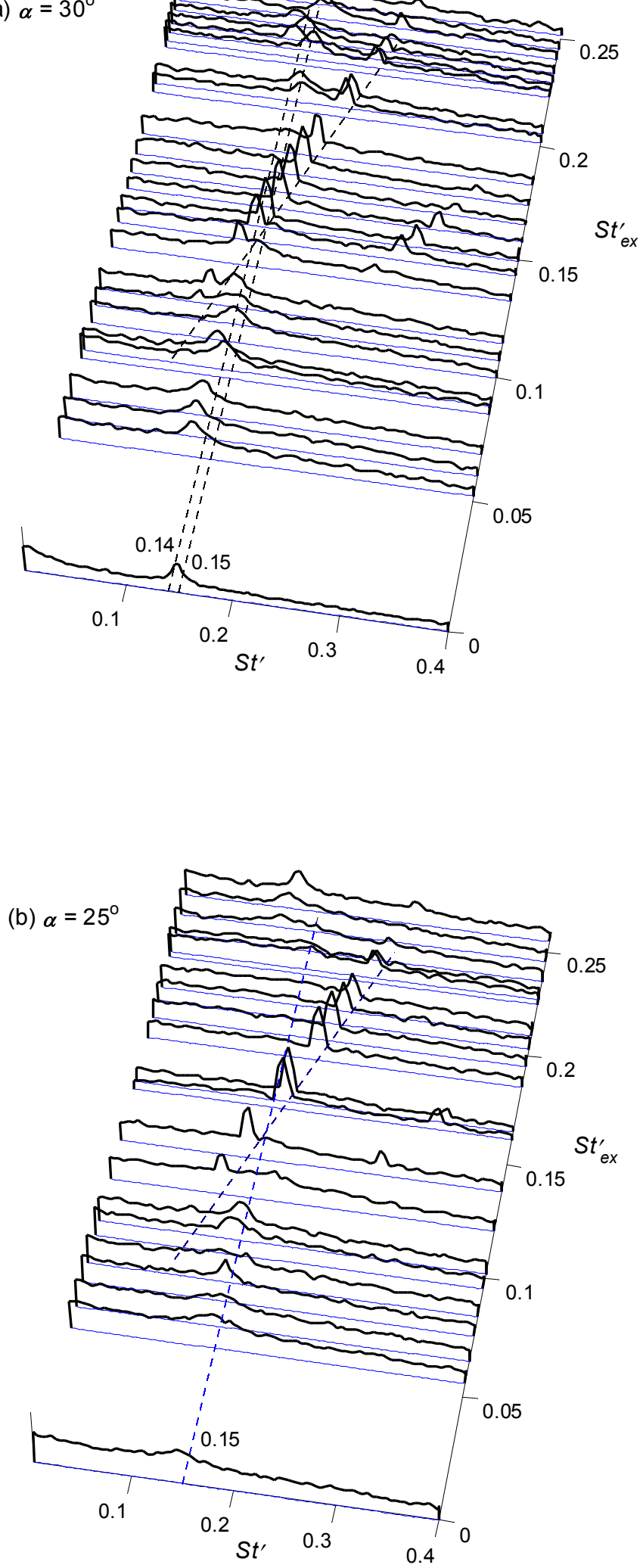
Lam and Leung, Figure 2

(c) $\alpha=20^{\circ}$
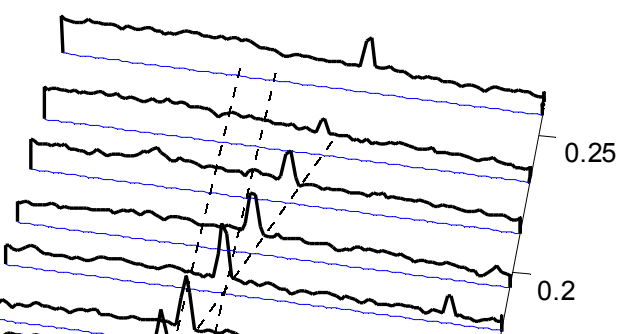

0.2
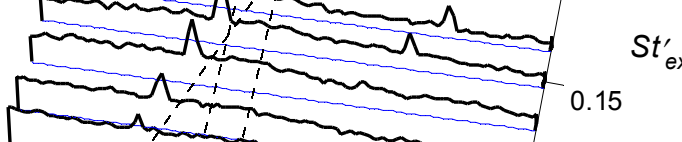

C
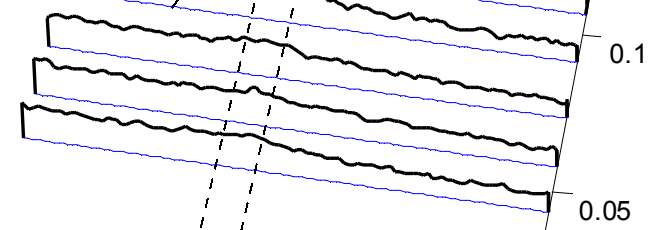

$\underbrace{0.15_{i}^{i}} \underbrace{i} 0.18$

0.1

0.2

$S t^{\prime} \quad 0.3$ 
Lam and Leung, Figure 3
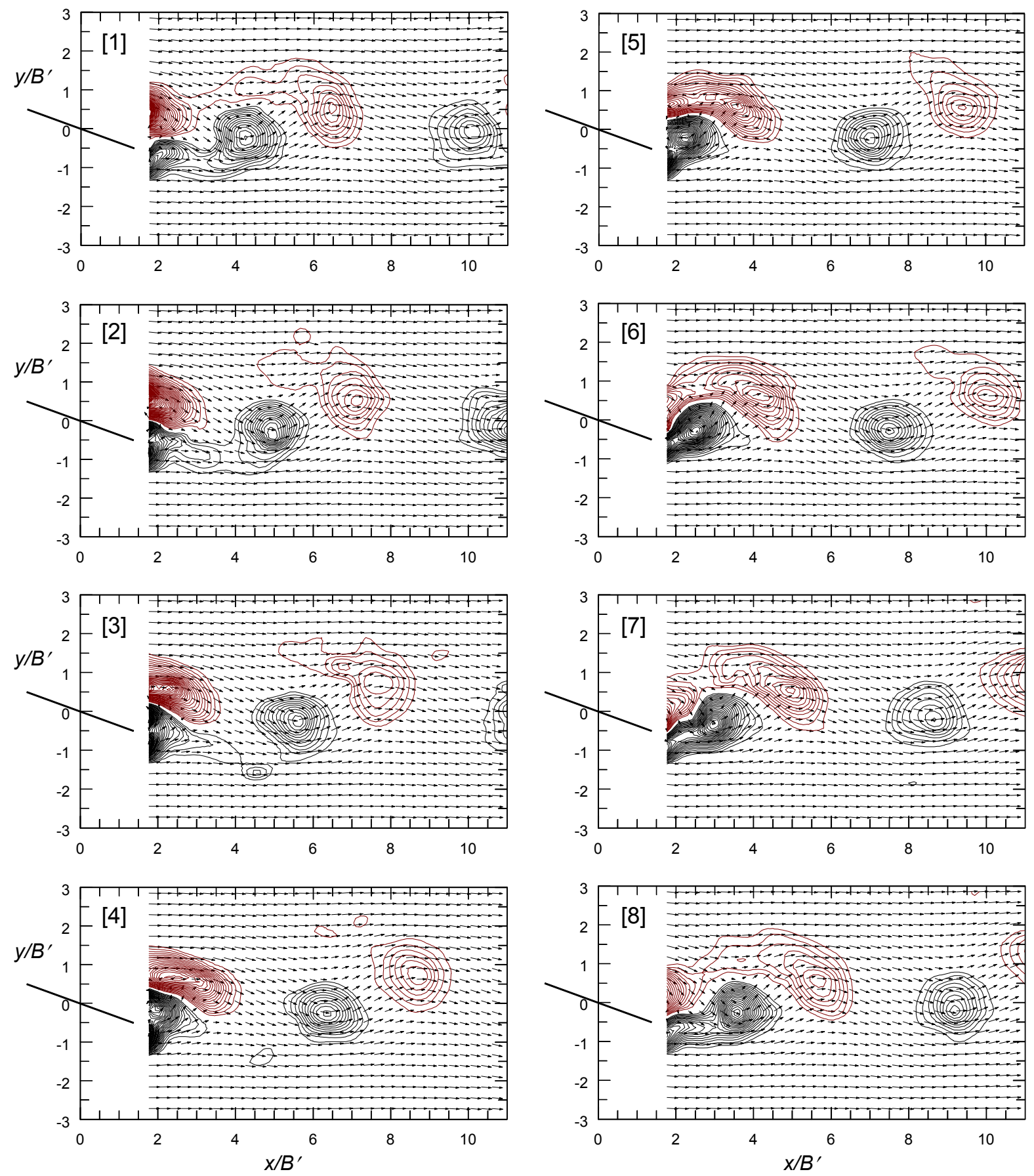


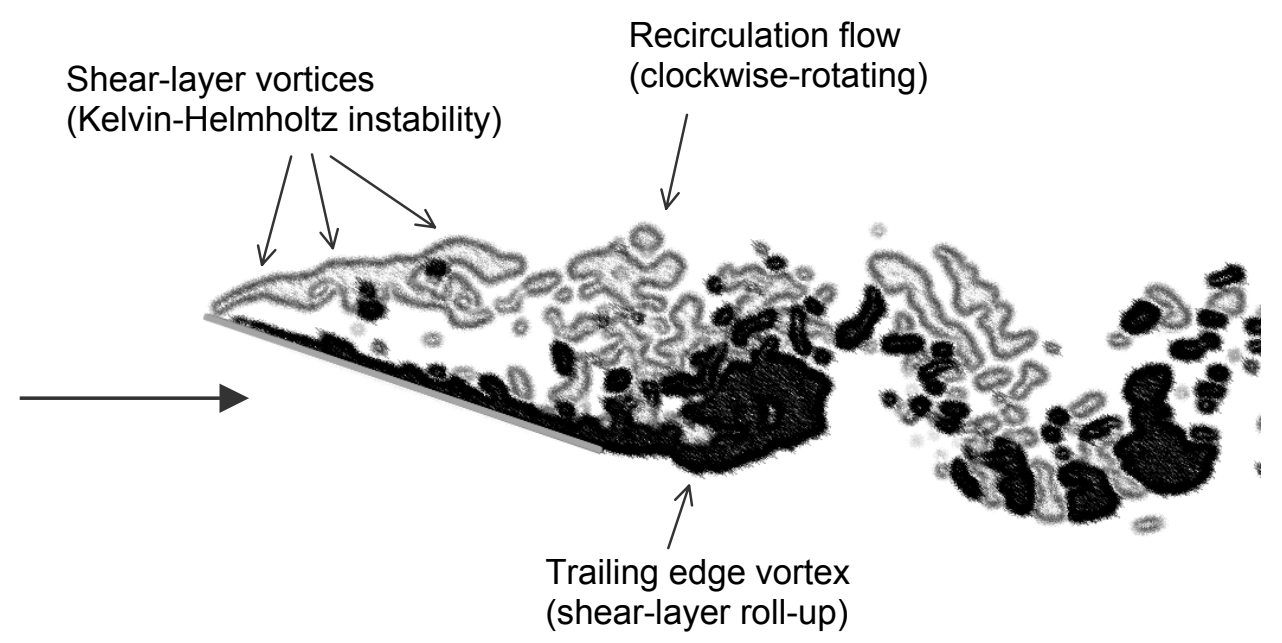


Lam and Leung, Figure 5

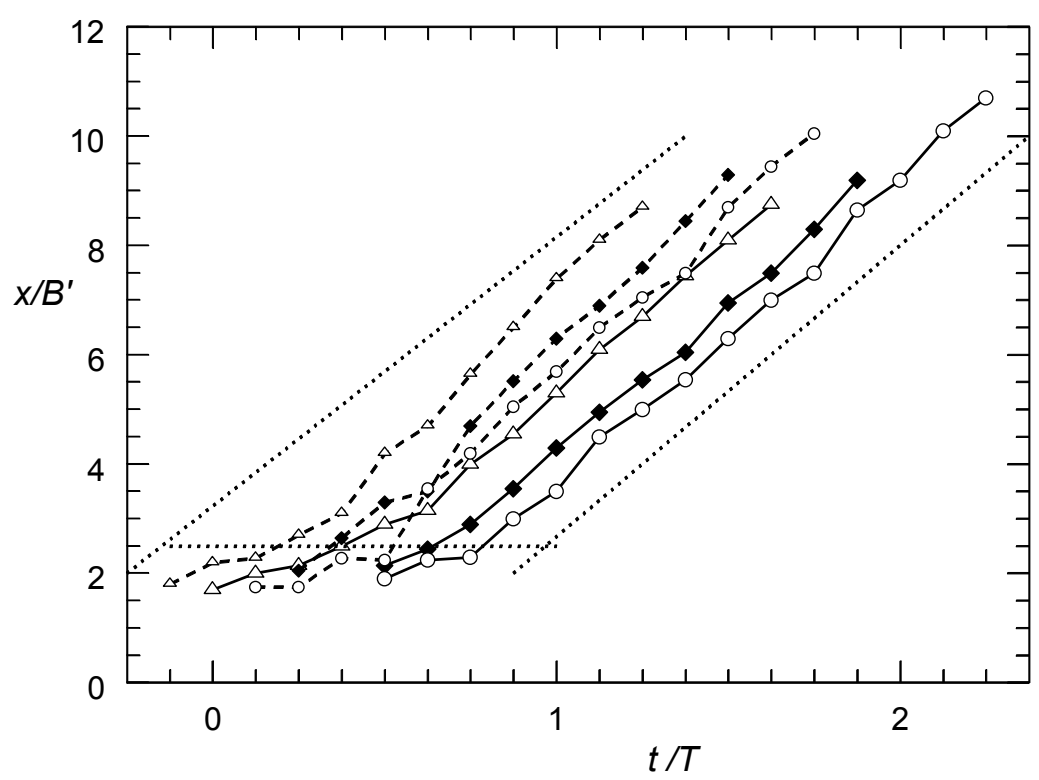


(a)
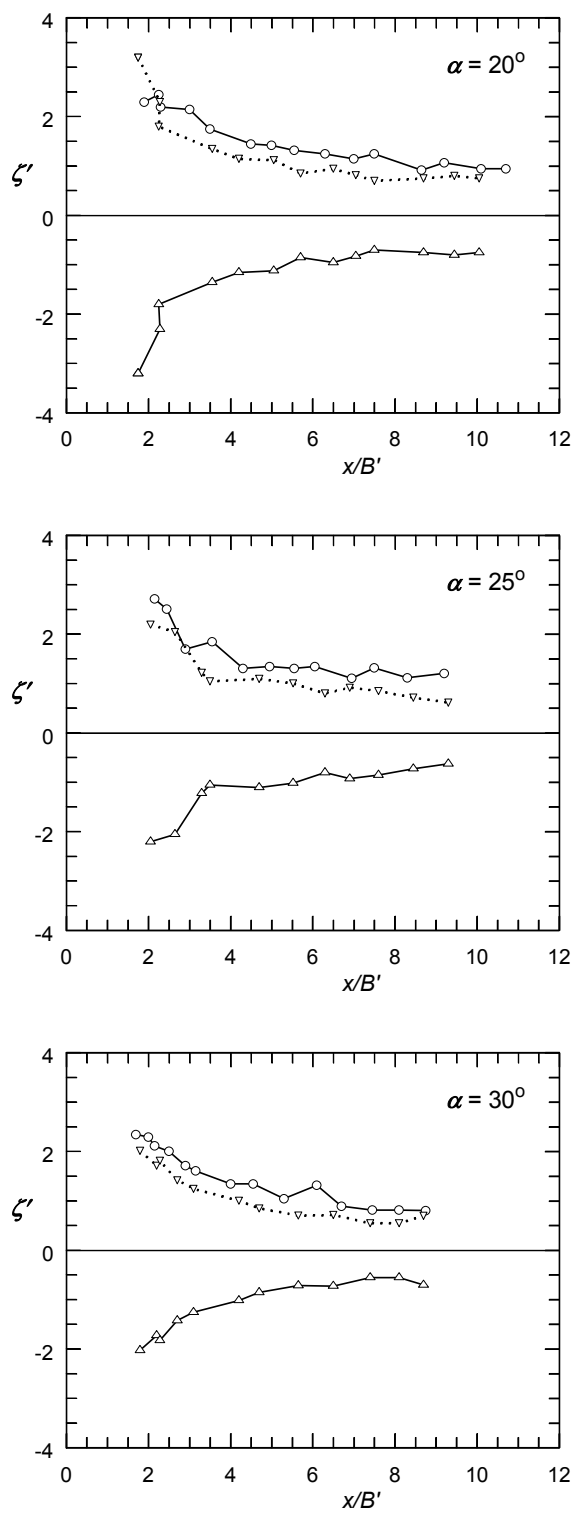

(b)
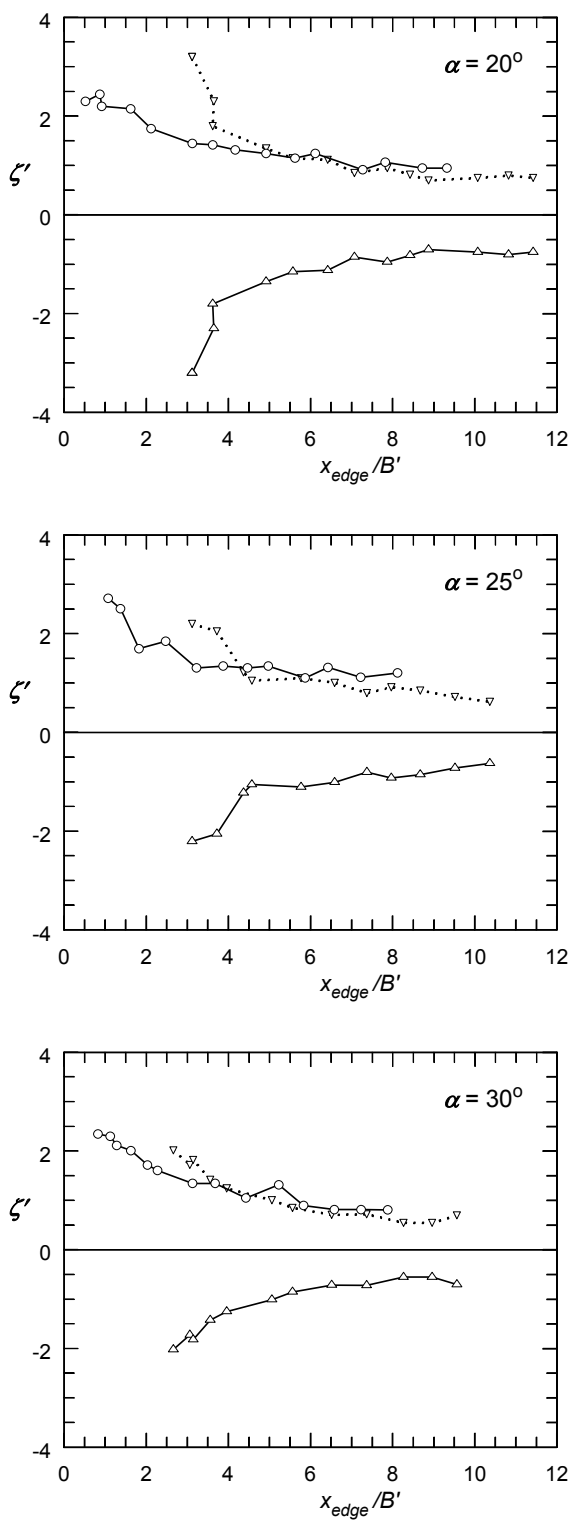
Lam and Leung, Figure7
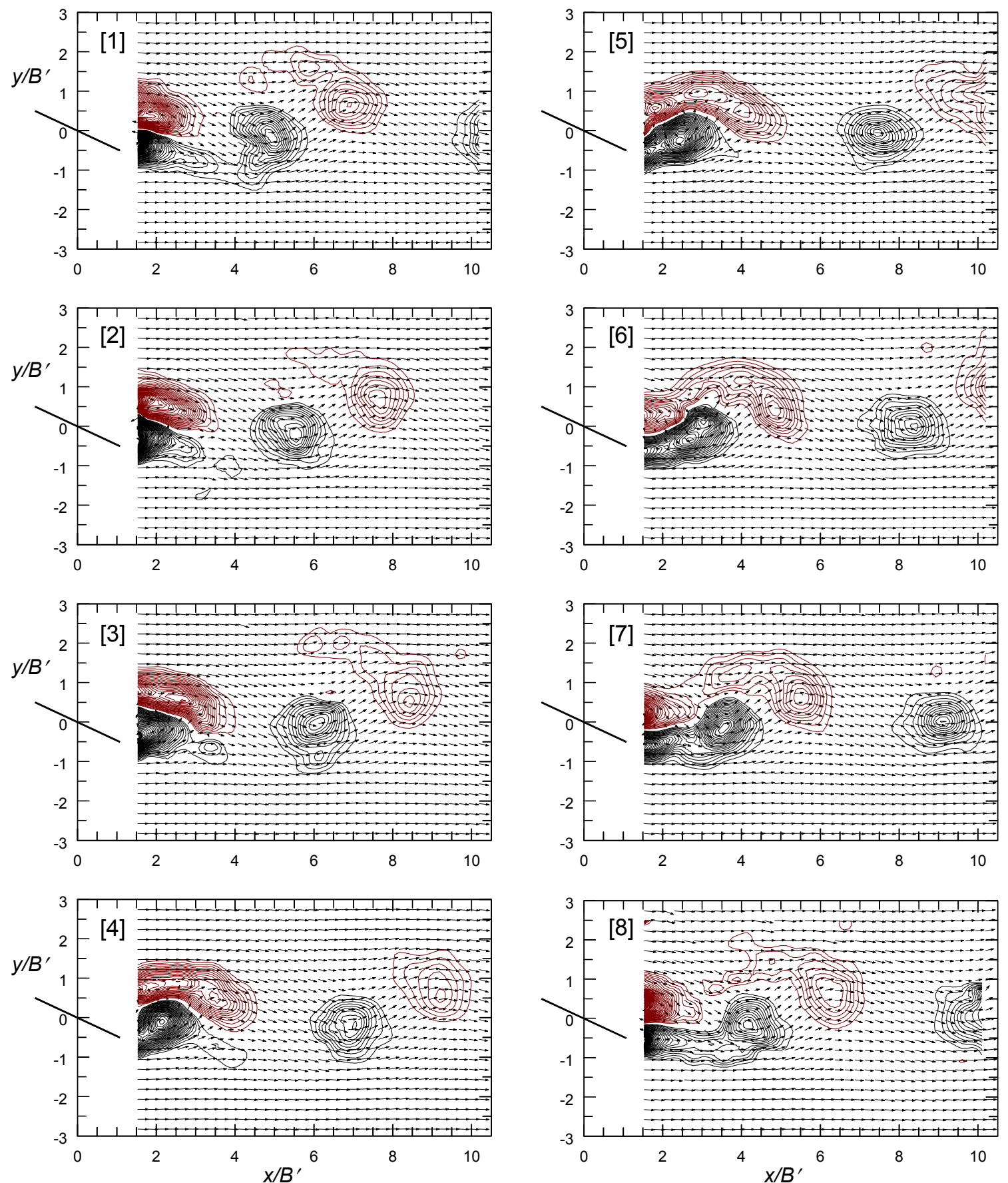

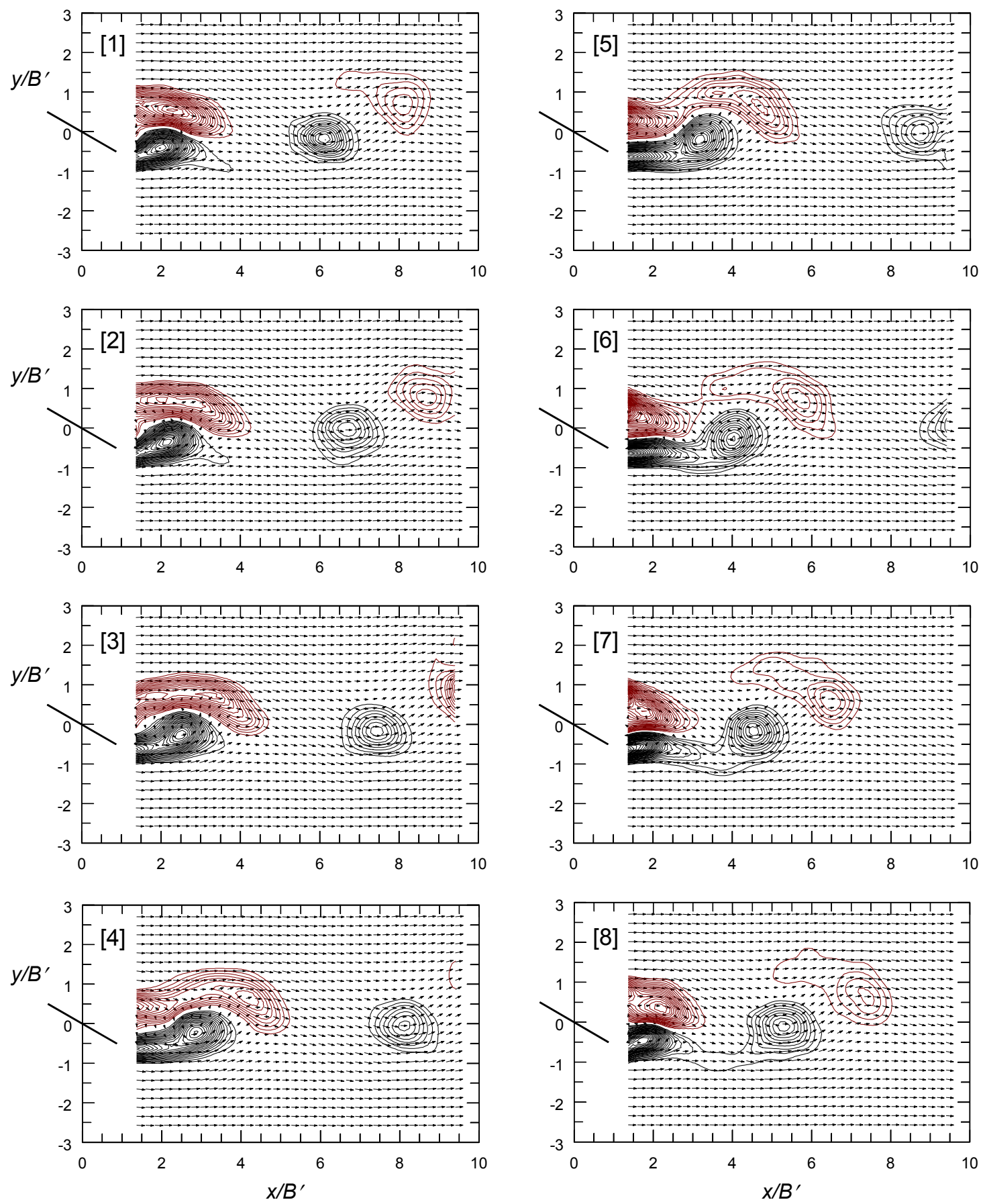
Lam and Leung, Figure 9
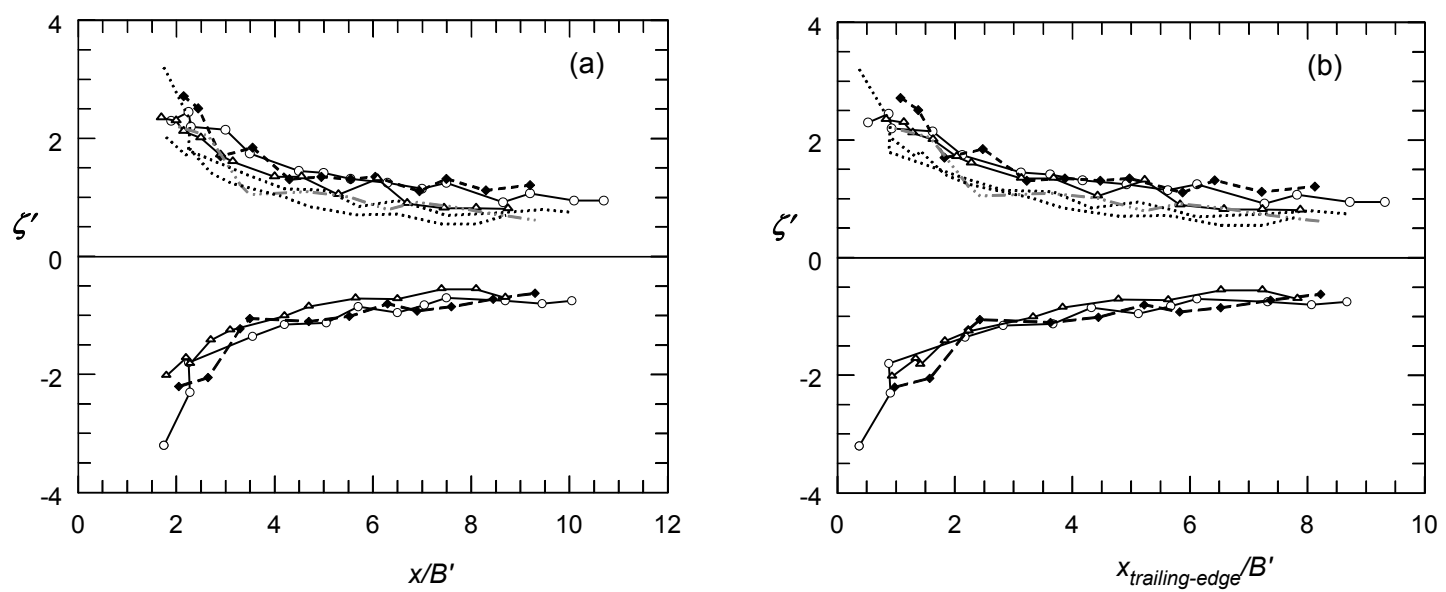
(a)
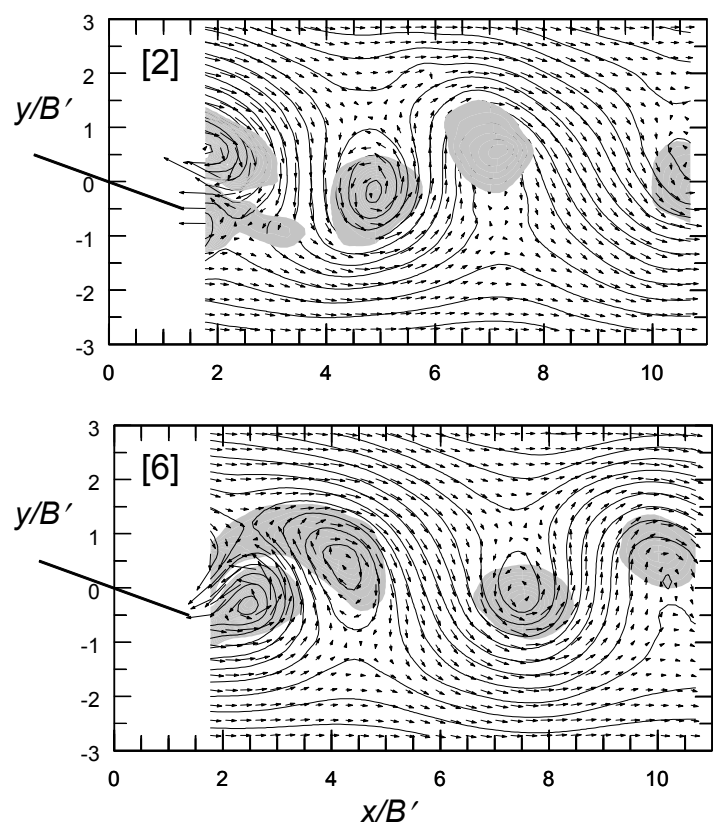

(c)
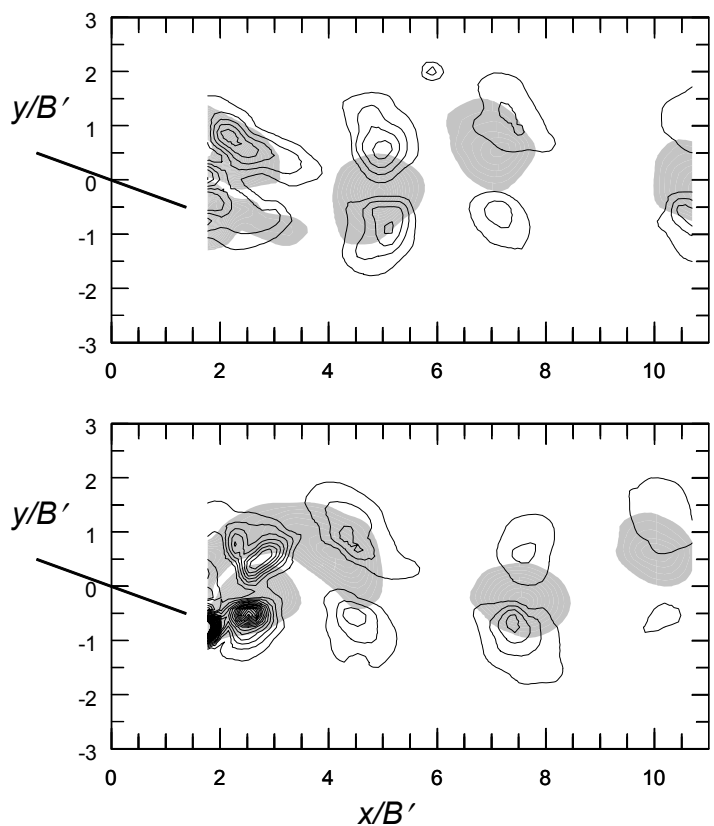

(b)
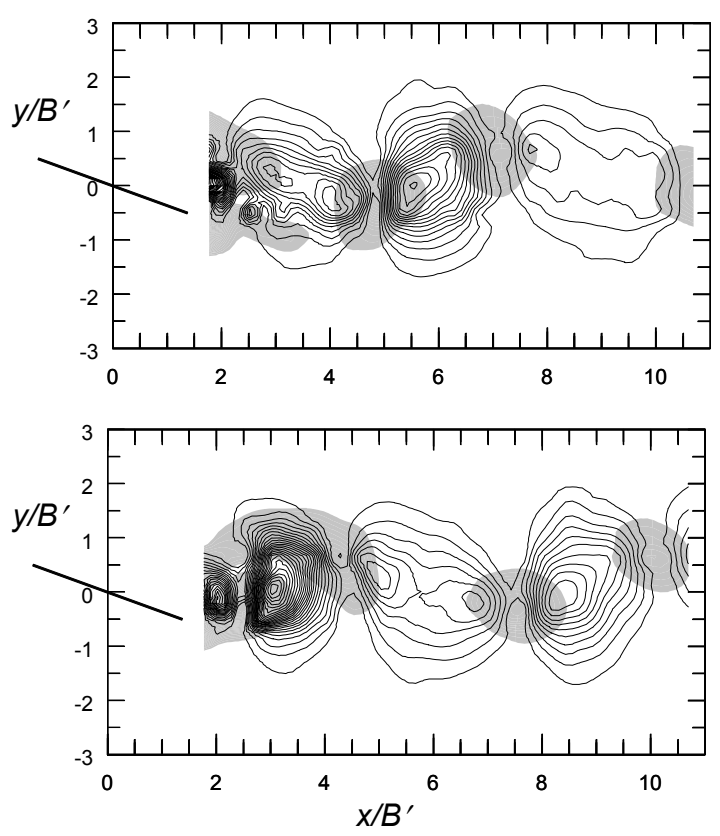

(d)
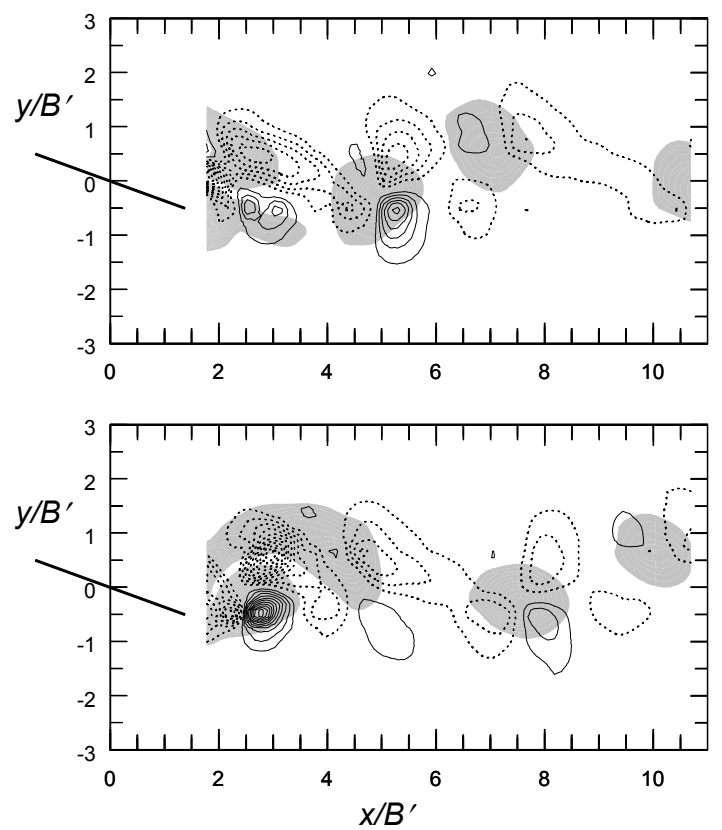
(a)
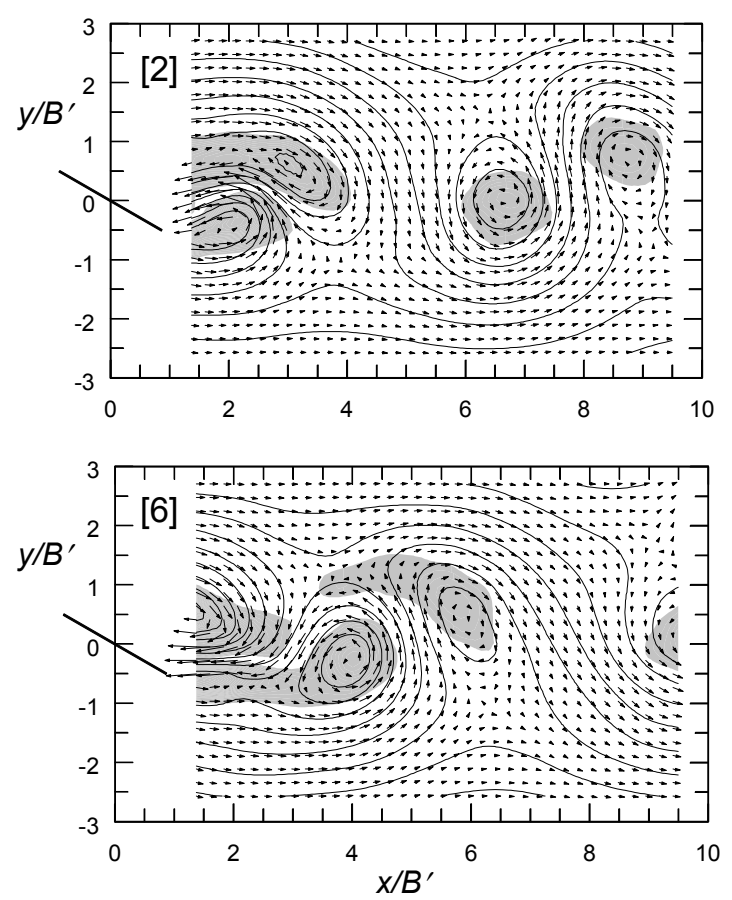

(c)
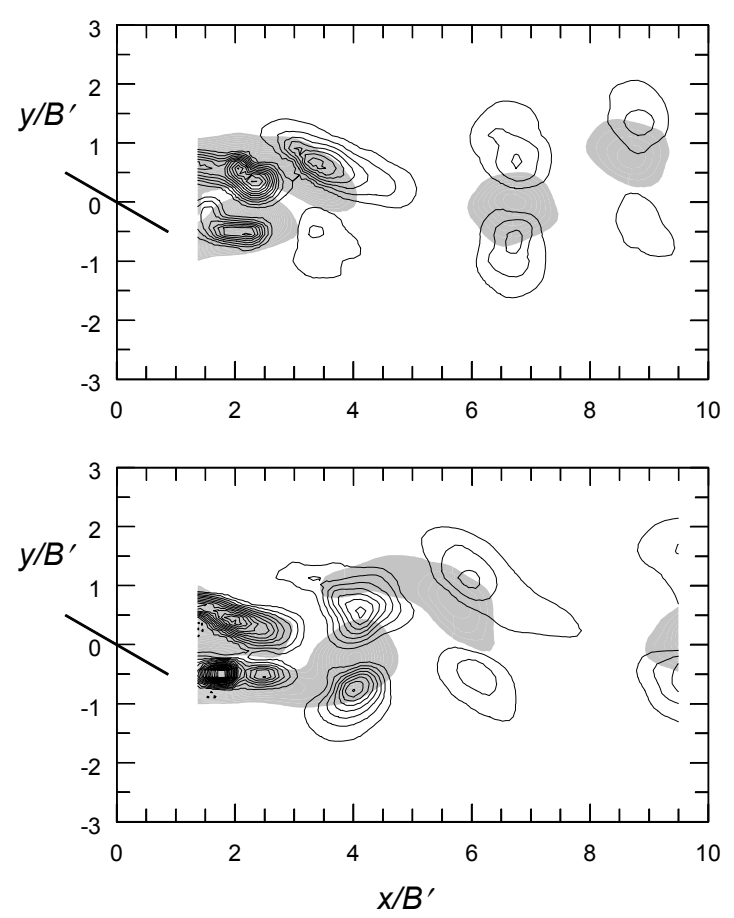

(b)
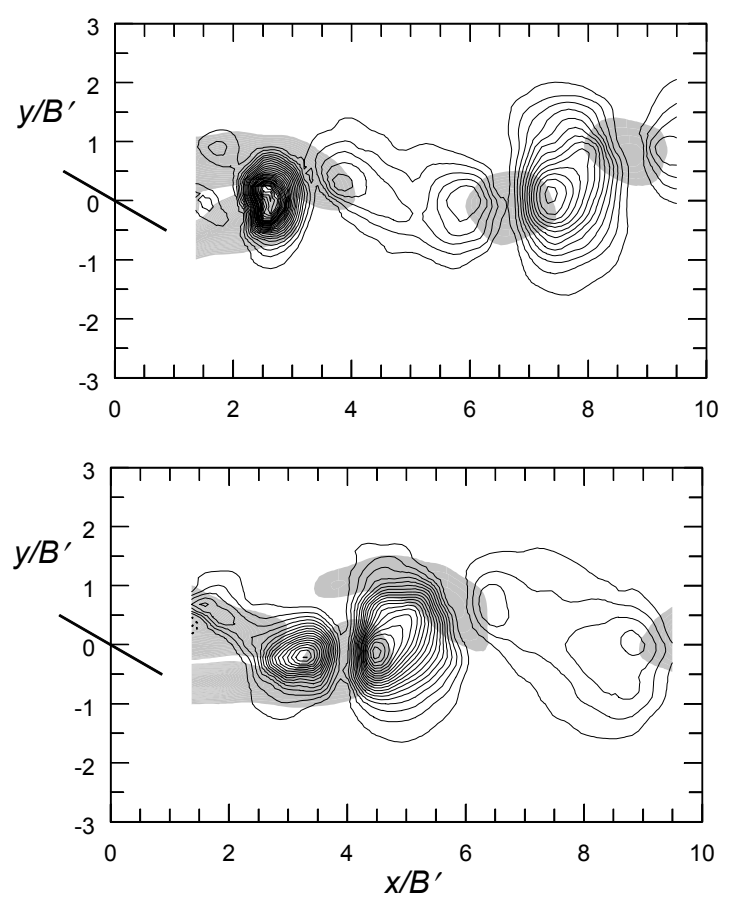

(d)
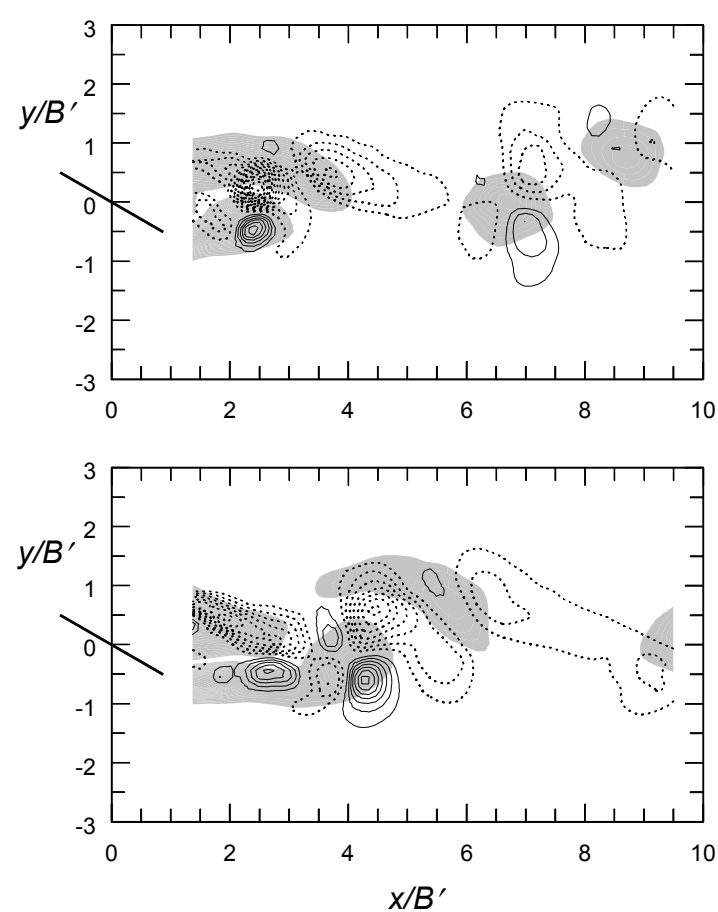